The Journal of $\mathbf{N}_{\text {onlinear }}$ S cience and Applications

http://www.tjnsa.com

\title{
SOME PROPERTIES OF $B$-CONVEXITY
}

\author{
HONGMIN SUO ${ }^{1,2, *}$ \\ Communicated by S. S. Chang
}

\begin{abstract}
In this paper, we give a characteristic of $B$-convexity structures of finite dimensional $B$-spaces: if a finite dimensional $B$-space has the weak selection property then its $B$-convexity structure satisfies $H$-condition. We also get some relationships among $B$-convexity structures, selection property and fixed point property. We show that in a compact convex subset of a finite dimensional $B$-space satisfying $H$-condition the weak selection property implies the fixed point property.
\end{abstract}

\section{INTRODUCTION AND PRELIMINARIES}

The convexity of space plays a very important role in fixed point theory and continuous selection theory. There were many works deal with various kinds of generalized, topological, or axiomatically defined convexities [1, 2, 3, 4]. Most of them were to establish various fixed point theorems and selection theorems in topological space without linear structure such as some generalizations of Brouwer fixed point theorem, Fan-Browder fixed point theorem and Michael selection theorem [2, 5, 6, 7, 8]. Recently, Briec[2] introduced the $B$-convexity by algebra borrows from topological ordered vector spaces and semilattice both. Briec proved that all the basic results related to fixed point theorems available in $B$ convexity[2].

The aim of this paper is to give some relationships among $B$-convexity structure, selection property and fixed point theorems. We prove that if $X$ is a $B$-space

Date: Received: 2 January 2009; Revised: 5 March 2009.

${ }^{*}$ Corresponding author. Supported by the Scientific Research Foundation of GuiZhou (No.2008(2050)).

2000 Mathematics Subject Classification. Primary 46A55; Secondary 47H10, 54H25.

Key words and phrases. B-Convexity, continuous selection, fixed point, $K K M$-maping. 
with $B$-convexity and of weak selection property with respect to any standard simplex $\Delta_{N}$ then $X$ satisfies $H$-condition, and we show that in a compact convex subset of a $B$-space with $B$-convexity structure the weak selection property implies the fixed point property.

A $B$-convex set can be seen as an abstract cone, in as much as we have a partial order and a multiplication by positive reals compatible with that partial order, we will remain in the finite dimensional setting of $R^{n}$ with its natural partial order. Let $n_{1}$ and $n_{2}$ be two positive integers whose sum is $n$ and

$$
\begin{array}{ll}
R_{-}^{n_{1}}=\left\{\left(x_{1}, \cdots, x_{n_{1}}\right) \in R^{n_{1}} \quad \max \left\{x_{i}\right\} \leq 0\right\}, \\
R_{+}^{n_{2}}=\left\{\left(x_{1}, \cdots, x_{\left.n_{2}\right)} \in R^{n_{2}} \quad \max \left\{x_{i}\right\} \leq 0\right\} .\right.
\end{array}
$$

We identify $R_{-}^{n_{1}} \times R_{+}^{n_{2}}$ with an octant of $R^{n}$. For $t \in R_{+}$and $x \in R_{-}^{n_{1}} \times R_{+}^{n_{2}}, t x$ is usual multiplication by a scalar, for $x$ and $y$ in $R_{-}^{n_{1}} \times R_{+}^{n_{2}}$, we let $x \vee y$ be the element of $R_{-}^{n_{1}} \times R_{+}^{n_{2}}$ defined in the following way:

$$
(x \vee y)_{j}=\left\{\begin{array}{cll}
\min \left\{x_{j}, y_{j}\right\} & \text { if } & j \leq n_{1} \\
\max \left\{x_{j}, y_{j}\right\} & \text { if } & j>n_{1} .
\end{array}\right.
$$

Then one can easily see that:

(A) $(x, y) \rightarrow x \vee y$ is associative, commutative, and idempotent, and also continuous, and $x \vee 0=x$ for all $R_{-}^{n_{1}} \times R_{+}^{n_{2}}$.

(B) For $t \in R_{+}$, the map $t \rightarrow t x$ is continuous and order preserving, and for all $t_{1}, t_{2}$ in $R_{+}$and for all $x$ and $y$ in $R_{-}^{n_{1}} \times R_{+}^{n_{2}},\left(t_{1} t_{2}\right) x=t_{1}\left(t_{2} x\right)$ and $t(x \vee y)=(t x) \vee(t y)$.

A finite dimensional $B$-space (of type $\left.\left(n_{1}, n_{2}\right)\right)$ ) is, by definition, a subset $X$ of $R_{-}^{n_{1}} \times R_{+}^{n_{2}}$ such that:

(BS) $0 \in X, \forall t \geq 0$ and $\forall x \in X, t x \in X$ and $\forall x, y \in X, x \vee y \in X$.

For a subset $B$ of $X$ the following properties are equivalent[1]:

(B1) $\forall x, y \in B, t x \vee y \in B \quad \forall t \in[0,1]$,

(B2) $\forall x_{1}, \cdots, x_{m} \in B$, and $\forall t_{1}, \cdots, t_{m} \in[0,1]$ such that

$$
\max _{1 \leq i \leq m}\left\{t_{j}\right\}=1, \quad t_{1} x_{1} \vee \cdots \vee t_{m} x_{m}=\vee t_{i} x_{i} \in B \text {. }
$$

Definition 1.1. A subset of $X$ for which (B1) or (B2) holds is called $B$-convex [1].

For example (B1) holds for increasing set ( $S$ is increasing if $x \leq y$ and $x \in S$ implies $y \in S)$. Sets of the form $\prod_{i=1}^{m}\left[a_{i}, b_{i}\right]$ are B-convex in $R_{+}^{n}$. 
Since an arbitrary intersection of $B$-convex sets is $B$-convex, and arbitrary set $S \subset X$ is always contained in a smallest $B$-convex subset of $X$, we call that set the $B$-convex hull of $S$, it is denoted by $[S]$. From (B2) one has the following characterization:

The $B$-convex hull of $S$ it is the set of all elements of the form $t_{1} x_{1} \vee \cdots \vee t_{m} x_{m}$ with $x_{i} \in S$ and $\max _{1 \leq i \leq m}\left\{t_{j}\right\}=1, t_{i} \in[0,1]$.

$B$-convex sets also are contractible[2]. We recall that a set $A$ is contractible if there exists a continuous map $h: A \times[0,1] \rightarrow A$ such that the map $a \rightarrow h(a, 0)$ is constant and $a \rightarrow h(a, 1)$ is the identity map of $A$.

For finite dimensional $B$-space $\mathrm{X}$ we define a map as follows:

$$
\left(K(x, y, t)=\left\{\begin{array}{c}
x \vee 2 t y \quad \text { if } \quad 0 \leq t \leq 1 / 2 \\
(2-2 t) x \vee y \quad \text { if } \quad 1 / 2<t \leq 1
\end{array}\right.\right.
$$

To see that a $B$-convex set $B$ is contractible one fixes $x_{0} \in B$ and take $h(x, t)=$ $K\left(x_{0}, x, t\right)$.

Other properties of $B$-convex and foxed points theorem and related matters in the framework of $B$-convexity see [2].

A topological space $X$ with a convexity structure $C$ (e.g. $B$-convexity) is said to be of weak selection property with respect to $S$ if every multivalued mapping $F: S \rightarrow 2^{X}$ admits a singlevalued continuous selection whenever $F$ is lower semicontinuous and nonempty closed convex valued. $(X, C)$ is said to be of weak selection property with respect to $S$ if $F: S \rightarrow 2^{X}$ admits a singlevalued continuous selection whenever $F$ is multivalued mapping with nonempty convex images and preimages relatively open in $X$ (i.e., $F(x)$ is convex for each $x \in S$ and $F^{-1}$ is open in $S)$. $X$ is said to be of fixed point property if every continuous selfmap $F$ on $X$ has a fixed point in $X$.

Let $N=\{0,1,2, \cdots, n\}, \triangle_{N}=e^{0} e^{1} \cdots e^{n}$ be the standard simplex of dimension $n$, where $\left\{e^{0} e^{1} \cdots e^{n}\right\}$ is the canonical basis of $R^{n+1}$, and for $J \subset N$, and $\triangle_{N}=c o\left\{e^{j}: j \in J\right\}$ be a face of $\triangle_{N}$. For each $x \in e^{0} e^{1} \cdots e^{n}$, there is a unique set of numbers $t_{0}, \cdots, t_{n}$ with, $\sum_{t=0}^{n} t_{i}=1, t_{i} \geq 0, i \in N$ such that $x=\sum_{i=0}^{n} t_{i} e^{i}$. The coefficients $t_{0}, \cdots, t_{n}$ are called the barycentric coordinates of $x$. Let

$$
\chi(v)=\left\{i: v=\sum_{i=0}^{n} t_{i} e^{i}, t_{i} \geq 0\right\} .
$$

Definition 1.2. Let $\left\{T_{i}: i \in I\right\}$ be some simplicial subdivision of standard simplex $\triangle_{N}=e^{0} e^{1} \cdots e^{n}, \nu$ denote the collection of all vertices of all subsimplexes in in the subdivision. A function $\lambda: \nu \rightarrow\{0,1, \cdots, n\}$ satisfying

$$
\lambda(v) \in \chi(v), \forall v \in \nu,
$$


is called a normal labeling of this subdivision. Moreover, $T_{i}$ is called a completely labeled subsimplex or completely labeled lattice if $T_{i}$ must have vertices with the completes set of labels: $0,1, \cdots n$.

Theorem 1.3. Let $\left\{T_{i}: i \in I\right\}$ be any simplicial subdivision of $\triangle_{N}$ and normally labeled by a function $\lambda$. Then there exist odd numbers of completely labeled subsimplexes of lattices in the subdivision with respect to the labeling function $\lambda$.

Last theorem is famous Sperner's lemma [3].

Theorem 1.4. Let $Y$ be a topological space. For each $J \subset N$, let $\Gamma_{J}$ be a nonempty contractible subset of $Y$. If $\emptyset \neq J \subset J^{\prime} \subset N$ implies $\Gamma_{J} \subset \Gamma_{J^{\prime}}$, then there exists a continuous mapping $f$ such that $F\left(\triangle_{J}\right) \subset \Gamma_{J}$ for each nonempty subset $J \subset N$.

This is Horvath' lemma [6, 7].

\section{MAin RESUlts}

According to Horvath's lemma, we call that a finite dimensional $B$-space satisfies $H$-condition if the $B$-convexity has the following property:

$(H)$ For each finite subset $\left\{y_{0}, y_{1}, \cdots, y_{n}\right\} \subset Y$, there exists a continuous mapping $f: \triangle_{N} \rightarrow\left[\left\{y_{0}, y_{1}, \cdots, y_{n}\right\}\right]$ such that $f\left(\triangle_{J}\right) \subset\left[y_{j}: j \in J\right]$ for each nonempty subset $J \subset N$.

Now, we first prove the crucial result of this section as below.

Theorem 2.1. If a finite dimensional B-space $Y$ with $B$-convexity is of weak selection property with respect to any standard simplex, then a finite dimensional $B$-space $Y$ satisfies $H$-condition.

Proof. . Let $A=\left\{y_{0}, y_{1}, \cdots, y_{n}\right\}$ be any finite subset of $Y, \triangle_{N}=e^{0} e^{1} \cdots e^{n}$ the standard simplex of dimension $n$. For each $J \subset N$ and each face $\triangle_{J}$ of $\triangle_{N}$, denote the interior of $\triangle_{J}$ by

$$
\triangle_{J}^{0}=\left\{v \in \triangle_{J}: \chi(v)=J\right\} .
$$

Define $T: \triangle_{N} \rightarrow 2^{Y}$ as follows:

$$
T(x)=\left[\left\{y_{j}: j \in \chi(x)\right\}\right], x \in \triangle_{N} .
$$

It is routinely to check that $T$ is with nonempty convex images and preimages relatively open in $\triangle_{N}$. In fact, for each $y \in Y$ and each $x \in T^{-1}(y)$, there is only one face $\triangle_{J}, J=\chi(x)$ such that $x \in \triangle_{J}^{0}$. So $x \notin \triangle_{J^{\prime}}$ for any face $\triangle_{J^{\prime}}$ not containing $\triangle_{J}$. For any $\triangle_{J^{\prime}} \supset \triangle_{J}$, there exists a neighborhood $O(x) \subset \triangle_{N}$ of $x$ such that $O(x) \bigcap \triangle_{J^{\prime}}=\emptyset$ as every face $\triangle_{J^{\prime}}$ is closed and the number of faces $\triangle_{N}$ of is finite. Therefore, for any $z \in O(x)$, any face $\triangle_{J^{\prime}}$ contains $z$ only if $\triangle_{J} \subset \triangle_{J^{\prime}}$. Then for each $z \in O(x), z \in \triangle_{\chi(z)}$ implies $\triangle_{\chi(z)} \supset \triangle_{J}$, So that $\chi(z) \supset J=\chi(x)$. It follow that $T(z) \supset T(x)$ for all $z \in O(x)$, and so $y \in T(x) \subset T(z)$, i.e., $z \in T^{-1}(y)$ for all $z \in O(x)$. . Hence $T^{-1}(y)$ is relatively 
open in $\triangle_{N}$.

In addition, it is obvious that $T$ is nonempty closed and convex. Since $Y$ is of selection property with respect to any standard simplex, there exists a singlevalued continuous mapping $f: \triangle_{N} \rightarrow Y$ such that $f(x) \in T(x)$ for all $x \in \triangle_{N}$. The definition of $T$ implies that $f\left(\triangle_{J}\right) \subset\left[\left\{y_{j}: j \in J\right\}\right]$ for each nonempty subset $J \subset N$, which complete the proof.

Corollary 2.2. . If a finite dimensional B-space $Y$ with $B$-convexity is of weak selection property with respect to any compact Hausdorff space, then a finite dimensional B-space $Y$ satisfies $H$-condition.

Proof. . It is immediate from Theorem 2.1.

Let $X$ be a subset of a finite dimensional $B$-space Y. A multivalued mapping $F: X \rightarrow 2^{Y}$ is called a $K K M$-mapping if $[A] \subset \bigcup_{x \in A} F(x)$ for each finite subset $A \subset X$.

Theorem 2.3. Let $X$ is subset of a finite dimensional $Y$ B-space satisfying $H$ condition and $F: Y \rightarrow 2^{X}$ is a KKM-mapping. If $F$ is closed-valued, then family $\{F(y): y \in Y\}$ has the finite intersection property.

Proof. . Let $\left\{y_{0}, y_{1}, \cdots, y_{n}\right\}$ be arbitrary finite subset of $X$. Since $Y$ satisfies H-condition, there exists a singlevalued continuous mapping $f: \triangle_{N} \rightarrow$ $\left[\left\{y_{0}, \cdots, y_{n}\right\}\right]$ such that $f\left(\triangle_{J}\right) \subset\left[\left\{y_{j}: j \in J\right\}\right]$ for each nonempty subset $j \subset N$.

For each $k \in\{1,2, \cdots\}$ and each $\varepsilon_{k}=1 / k \geq 0$, let $\left\{T_{i}^{k}: i \in I_{k}\right\}$ be some simplicial subdivision of $\triangle_{N}$ such that the mesh of the subdivision less than $1 / 2^{k}$. And let $\nu^{k}$ be the set of vertices of all subsimplexes in this subdivision.

For each $v \in \nu^{k}$, let

$$
\lambda^{k}(v)=\min \left\{j \in \chi(v): f(v) \in F\left(y_{j}\right)\right\} .
$$

Then $\lambda^{k}(v)$ is nonempty, since $v \in \operatorname{conv}\left\{e^{j}: j \in \chi(v)\right\}$ and

$$
f(v) \in f\left(\left[\left\{e^{j}: j \in \chi(v)\right\}\right]\right) \subset\left[\left\{y_{j}: j \in \chi(v)\right\}\right] \subset \bigcup_{j \in \chi(v)} F\left(y_{j}\right) .
$$

By the hypothesis, it is easy to see that $\lambda^{k}$ is a normal label function of the subdivision.

So for each $k=1,2, \cdots$, there must exist a subsimplex $T_{i_{k}}$ with complete labels by Sperner's Lemma. Let $z_{0}^{k}, \cdots, z_{n}^{k}$ be all vertices of subsimplex $T_{i_{k}}$, and

$$
\lambda\left(z_{0}^{k}\right)=0, \lambda\left(z_{1}^{k}\right)=1, \cdots, \lambda\left(z_{n}^{k}\right)=n .
$$

By the definition of $\lambda$, we have

$$
f\left(z_{0}^{k}\right) \in F\left(y_{0}\right), f\left(z_{1}^{k}\right) \in F\left(y_{1}\right), \cdots, f\left(z_{n}^{k}\right) \in F\left(y_{n}\right) .
$$

Note that $z_{0}^{k}, \cdots, z_{n}^{k}$ are some vertices of subsimplex $T_{i_{k}}$, so that $d\left(z_{i}^{k}, z_{j}^{k}\right) \leq$ $1 / 2^{k}, i, j \in\{0,1, \cdots, n\}$. Since $\triangle_{N}$ is compact, we may assume that there 
is $y^{*} \in \triangle_{N}$ such that $z_{i}^{k} \rightarrow y^{*}, i=0,1, \cdots, n$. Then $f\left(z_{i}^{k}\right) \rightarrow f\left(y^{*}\right)$. It follows from the closeness of each $F\left(y_{i}\right)$ that $f\left(y^{*}\right) \in F\left(y_{i}\right), i=0,1, \cdots, n$, and $\bigcap_{i \in N} F\left(y_{i}\right) \neq \emptyset$. This completes the proof.

Theorem 2.4. . Let a finite dimensional B-space $Y$ satisfying $H$-condition, $X$ is a convex compact subset of $Y$, and $F: X \rightarrow 2^{X}$ a multivalued mapping with nonempty convex images and preimages relatively open in $X$. Then $F$ has a fixed point.

Proof. . Since $X$ is compact and $X=\bigcup_{x \in X} F^{-1}(x)$, there exists a finite subset $\left\{x_{0}, x_{1}, \cdots, x_{n}\right\}$ of $X$ such that $X=\bigcup_{i=0}^{n} F^{-1}\left(x_{i}\right)$. Then $\bigcap_{i=0}^{n}\left[X \backslash F^{-1}\left(x_{i}\right)\right]=\emptyset$. Let

$$
G(x)=\left[X \backslash F^{-1}(x)\right], \quad \forall x \in X .
$$

With Theorem 2.3, we know that $G$ is not a $K K M$-mapping, so that there exists a finite subset $\left\{y_{0}, y_{1}, \cdots, y_{n}\right\}$ such that

$$
\left[\left\{y_{0}, y_{1}, \cdots, y_{n}\right\}\right] \not \subset \bigcup_{i=0}^{m} G\left(y_{i}\right) .
$$

Then there is some $y^{*} \in\left[\left\{y_{0}, y_{1}, \cdots, y_{n}\right\}\right]$ such that $y^{*} \notin G\left(y_{i}\right)$ for all $i=$ $0,1, \cdots m$, that is

$$
y^{*} \in F^{-1}\left(y_{i}\right), \quad \forall i=0,1, \cdots, m \text {. }
$$

Consequently

$$
y^{i} \in F^{*}(y), \quad \forall i=0,1, \cdots, m
$$

Therefore

$$
y^{*} \in\left[\left\{y_{0}, y_{1}, \cdots, y_{m}\right\}\right] \subset F\left(y^{*}\right) .
$$

Which complete the proof.

Theorem 2.5. . Let $X$ be a compact topological space, a finite dimensional $B$ space $Y$ satisfying $H$-condition, and $F: X \rightarrow 2^{Y}$ a multivalued mapping with nonempty convex images and preimages relatively open in $X$. Then $F$ has a continuous selection.

Proof. . Since $X$ is compact and $X=\bigcup_{y \in Y} F^{-1}(y)$, there exists a finite subset $\left\{y_{0}, y_{1}, \cdots, y_{m}\right\}$ of $X$ such that $X=\bigcup_{i=0}^{n} F^{-1}\left(y_{i}\right)$. Now let $\left\{p_{i}: i=\right.$ $0,1, \cdots, n\}$ be a partition of unity subordinate to the finite covering $\left\{F^{-1}\left(y_{i}\right)\right.$ : $i=0,1, \cdots, n\}$. Define a mapping $\phi: X \rightarrow \triangle_{N}$ by

$$
\phi(x)=\sum_{i=0}^{n} p_{i}(x) e^{i}, \quad \forall x \in X .
$$

On the other hand, since $Y$ satisfies $H$-condition, there exists a singlevalued continuous mapping $f: \triangle_{N} \rightarrow\left[\left\{y_{0}, y_{1}, \cdots, y_{n}\right\}\right]$ such that $s\left(\triangle_{J}\right) \subset\left[y_{j}: j \in J\right]$ for each nonempty subset $J \subset N$.

Now our desired mapping $g$ is given by

$$
g=f \circ \phi
$$


In fact, it is easy to verify that $\phi(x) \in \triangle_{J(x)}$ for each $x \in X$, where $J(x)=\{i \in$ $\left.N: p_{i}(x) \neq 0\right\}$. By the convexity of $F(x)$, we do have that $\left\{y_{j}: J(x)\right\} \subset F(x)$ and thus

$g(x)=f(\phi(x)) \subset f\left(\triangle_{J(x)}\right) \subset\left[y_{j}: j \in J\right] \subset\left[y_{j}, p_{j}(x) \neq 0\right] \subset\left[y_{j}: y_{j} \in F(x)\right] \subset F(x)$.

This complete the proof.

\section{REFERENCES}

1. W. Briec and C.D. Horvath, B-convexity, Optimization. 53 (2004), 103-127.

2. W. Briec and C.D. Horvath, Nash points, Ky Fan inequality and equilibria of abstract economies in Max-Plus and B-convexity, J. Math. Anal. Appl. Vol 341,Issue 1 (2008), 188199.

3. M. van de Vel, Theory of Convex Structures, North-Holland Mathematical Library. Vol.50, North-Holland Publishing Co., Amsterdam, 1993. 1, 1, 1.1.

4. C.D. Horvath, Contractibility and general convexity, J. Math. Anal. Appl, 156 (1991), 341-357. 1, 1, 1 ,

5. S. Park, Fixed point theorems in locally G-convex spaces, Nonlinear Anal., 48 (2002), 868-879. 1, 1

6. X.P. Ding and W.A. Kim and K.K. Tan, A New minimax inequality on G-spaces with applications,Bull. Austra. Math. Soc. 41 (1990), 457-473. 1

7. C. Horvath, Some results on multivalued mappings and inequalities without convexity, in nonlinear and convex analysis,(Ed.B.L.Lin and S.Simons ), Lecture Notes in Pure and Applied Math,Marcel Dekker 1987, 99-106. 1

8. S.-S. Chang, G. X.-Z. Yuan, G.-M. Lee, and Xiao-Lan Zhang, , Saddle points and minimax theorems for vector-valued multifunctions on $H$-spaces. Appl. Math. Lett. 11 (1998), 101107. 1 , 1 ,

1. 1.

${ }^{1}$ School of Mathematics and Computer Science, GuiZhou University for NAtionalities , 550025, Guiyang, Guizhou, China.

${ }^{2}$ School of Mathematics and Statistics, Southwest University, 400715, Chongqing, CHINA.

E-mail address: gzmysxx88@sina.com 